\title{
Introduction of Progress in Education under Recent Technology Revolution
}

\author{
Chengyan $\mathrm{Li}^{1} \cdot$ Xin $\mathrm{Gao}^{2} \cdot$ Qingquan Sun ${ }^{3}$ \\ Published online: 5 February 2021 \\ (C) The Author(s), under exclusive licence to Springer Science+Business Media, LLC part of Springer Nature 2021, corrected publication 2021
}

Nowadays, the new technology revolution brings a fast progress for pedagogy. The popularization of cloud computing, edge computing and $5 \mathrm{G}$ network provides a new opportunity for the development of mobile education, which makes the lifelong education and fragmented education possible. Meantime, the worldwide use of artificial intelligence (AI) and big data technology are changing research domain of educational technology, and will bring novel study in individualized education and teaching reformation. So, there are many remaining scientific and technical problems in it. For example, the use of edge computing pattern and multi-modal information in education, the application of $\mathrm{AI}$ and big data technology in teaching reformation, image information extraction from user's handwritten manuscripts, and the stability of cloud platform of mobile education, etc. Meantime, emerging methods which can improve the efficiency of this domain are also welcome.

In order to provide an opportunity for researchers to publish their gifted studies of theory and technology in this research domain, this half theme issue is proposed to collect advanced method in Education under Recent Technology Revolution, as well as excellent engineering applications within this domain. With support of eLEOT 2019, in this half theme issue, 6 in 16 submissions are accepted with ratio $37.5 \%$. In these 6 papers, 3 papers come from China, others are from the United States and Australia, respectively.

The first article "Design and Research of Intelligent Question-Answering(Q\&A) System Based on High School

Qingquan Sun

qsun@csusb.edu

1 School of Computer Science and Technology, Harbin University of Science and Technology, Harbin, China

2 Computer, Electrical and Mathematical Sciences and Engineering Division, King Abdullah University of Science and Technology, Jeddah, Saudi Arabia

3 Department of Computer Science, California State University San Bernardino, San Bernardino, CA, USA
Course Knowledge Graph" is authored by Zhijun Yang from Educational Department of Yunnan Province and Hang Li from the University of Queensland, Australia. This article focuses on question answering which is an indispensable link in high school teaching. Through question answering, students' learning doubts can be solved and teaching feedback can be acquired. However, through the investigation and research, it is found that the effect of question answering is not satisfactory with the expansion of student size [1].

Therefore, their article analyzes the current situation of question answering and designs an intelligent question answering system based on constructivism learning theory and cognitive structure learning theory. The system, which is the first innovative application in the field of high school teaching, integrates knowledge graph technology, intelligent question answering technology and big data technology. It can solve students' questions in time and accurately, link the knowledge points to the questions, help students construct knowledge network graph, analyze students' questioning behavior and predict their learning behavior.

The second article "A Survey of CRF Algorithm based Knowledge Extraction of Elementary Mathematics in Chinese" is authored by Shuai Liu from Hunan Provincial Key Laboratory of Intelligent Computing and Language Information Processing, Hunan Normal University. Online education is more and more popular under the influence of the epidemic. Information extraction is a fundamental technique which is utilized to support online education. Chinese word segmentation is an important research issue in elementary mathematics knowledge extraction. The speed of segmentation directly affects subsequent applications, and the accuracy of segmentation directly affects corresponding research in the next step. Conditional Random Field (CRF) model that is effective to discover new word is increasingly used in knowledge extraction of basic mathematics [2].

This article firstly introduces the traditional CRF process of named entity recognition. Then, an improved algorithm named CRF++ is proposed. Since the recognition rate of named entities based on traditional machine learning methods 
is not good enough, a post-processing method for entity recognition that automatically generates a dictionary is proposed. After identifying mathematical entities, a pruning strategy combining Viterbi algorithm and rules is proposed to achieve a higher recognition rate of elementary mathematical entities. Finally, several methods of disambiguation are introduced.

The third article "Video Question Answering: A Survey of Models and Datasets" is authored by Guanglu Sun from the School of Computer Science and Technology, Harbin University of Science and Technology, China. Video question answering (VideoQA) automatically answers natural language question according to the content of videos. It helps the effective aggregation of massive data and provides educational resources for online learning, so it promotes the development of online education, scenario analysis, etc. VideoQA is a challenging task because it requires a model to understand multi-modal semantic information of the video and the question to generate the answer [3].

Firstly, this article proposes a general framework of VideoQA which consists of a video feature extraction module, a text feature extraction module, an integration module, and an answer generation module. The integration module is the core module, including core processing model, recurrent neural networks (RNNs) encoder and feature fusion. These three submodules cooperate to generate the contextual representation, and the answer generation module generates the answer based on it. Then, it summarizes the methods in core processing mod$\mathrm{el}$, and introduces the ideas and applications of the methods in detail, such as encoder-decoder, attention model, and memory network and other methods. Additionally, the widely used datasets and evaluation criteria are introduced, as well as the analysis of experimental results on benchmark datasets.

The fourth article "“" 3 M" Performability Creative Practical Platform Application Research" is authored by Tiejun Zhu from Anhui Polytechnic University, China and Ablaye Camara from University of Detroit Mercy, USA. The design theory course has not undergone any major reforms in recent years to keep pace with new technology, modern teaching methodologies or student expectations. It is mainly due to the traditional approach of emphasizing practice rather than design theory being inherent in design education in China. The key to solve this problem is to effectively link the design theory course to universal practice. Therefore, endogenous, autonomous, targeted and unique design theory course practice resources and platforms are extremely important [4].

This article empirically takes the design theory course of "Advertising Copywriting" as the case study and introduces Micro-lecture. It is advocated by China's Ministry of Education to achieve accurate imparting of core knowledge points of the design theoretical contents. At the same time, it combines five new stratified progressive multiple teaching approaches namely: meta-cognitive, self-inspiration, research-discussion, flipped classroom and scholarly exploration to effectively improve the course teaching form and enrich classroom activities. In addition, this article recommends the implementation of the "Maker" practice including real time projects and training Maker ideology.

The fifth article "Research on Optimal CheckpointingInterval for Flink Stream Processing Applications" is authored by Zhan Zhang from Harbin Institute of Technology, China. Nowadays, various distributed stream processing systems (DSPSs) are employed to process the ever-expanding realtime data. The DSPSs are highly susceptible to system failure, and the fault-tolerance issue is a major problem, which is drawing lot of attention. Flink is a popular streaming computing framework that implements a lightweight, asynchronous checkpoint technique based on the barrier mechanism to ensure high efficiency in analyzing data. In a checkpoint-based fault-tolerance mechanism, a shorter checkpoint interval can increase runtime cost of streaming applications, while a longer one will increase recovery time of failure recovery. So, selecting an optimal checkpoint interval is critical to attain high efficiency of the streaming applications. Traditional optimal checkpoint interval mechanisms usually assume that the checkpointing delay and the fault recovery time are fixed. However, both factors have a strong relation to the intensity of the application's workload. [5].

To obtain more optimal checkpoint interval under different workload intensities, this article proposes a performance model to estimate the tuples processing latency and a recovery model to estimate the fault recovery time. With these two models, an optimal checkpoint interval can be arrived at. These models and the interval optimization interval are verified experimentally on Flink. The results show that the proposed model can recommend an optimal checkpoint interval according to the system reliability related indicators. This proposed system optimized recovery time and performs efficiently in applications with delay constraints.

The sixth article "Research on Image Fusion Algorithm Based on NSST Frequency Division and Improved LSCN" is authored by Hongna Zhang from the College of Mechanical Engineering, Inner Mongolia University for the Nationalities, China and Wei Yan from the College of Mechanical Engineering, University of Florida, U.S.A. With the continuous development of science and technology, there are many types of medical images for reference in clinical diagnosis. The information of human tissues and organs can be reflected in different modal medical images. Although single-modal images have unique advantages in representing characteristic information of human tissues, it provides limited information and cannot reflect all the details of the relevant tissues, which leads to misdiagnosis in clinical medicine. Therefore, medical images need multimodal fusion to compensate for the lack of information in a single mode [6].

This article proposes a medical image fusion algorithm based on non-down-sampling shear wave transform (NSST). This algorithm fuses multi-modal medical images, enriches 
the information of fused images, improves the image quality, and provides a basis for clinical diagnosis. The proposed algorithm can obtain better fusion effects in both objective and subjective evaluation. The obtained fusion image has rich information, excellent edge retention characteristics, subjectively clear texture and high contrast and good visual effect.

Acknowledgements The guest editors are thankful to our reviewers for their effort in reviewing these manuscripts. We also thank the Edit-inChief, Dr. Imrich Chlamtac for his supportive guidance during the entire process. The editorial is supported by National Natural Science Foundation of China (Grant No. 61702140), The Scientific Research Foundation for the Overseas Returning Person of Heilongjiang Province of China (LC2018030), and the Fundamental Research Foundation for Universities of Heilongjiang Province (JMRH2018XM04).

\section{References}

1. Yang Z, Wang Y, Gan J et al (2021) Design and research of intelligent Question-Answering(Q\&A) system based on high school course knowledge graph. Mobile Netw Appl. https://doi.org/10. 1007/s11036-020-01726-w

2. Liu S, He T, Dai J (2021) A survey of CRF algorithm based knowledge extraction of elementary mathematics in Chinese. Mobile Netw Appl. https://doi.org/10.1007/s11036-020-01725-x

3. Sun G, Liang L, Li T et al (2021) Video question answering: a survey of models and datasets. Mobile Netw Appl. https://doi.org/10.1007/ s11036-020-01730-0

4. Tiejun Z, Camara A (2021) " 3 M" performability creative practical platform application research. Mobile Netw Appl. https://doi.org/10. 1007/s11036-020-01727-9

5. Zhang Z, Li W, Qing X et al (2021) Research on optimal checkpointing-interval for flink stream processing applications. Mobile Netw Appl. https://doi.org/10.1007/s11036-020-01729-7

6. Zhang H, Yan W, Zhang C et al (2021) Research on image fusion algorithm based on NSST frequency division and improved LSCN. Mobile Netw Appl. https://doi.org/10.1007/s11036-020-01728-8

Publisher's note Springer Nature remains neutral with regard to jurisdictional claims in published maps and institutional affiliations.

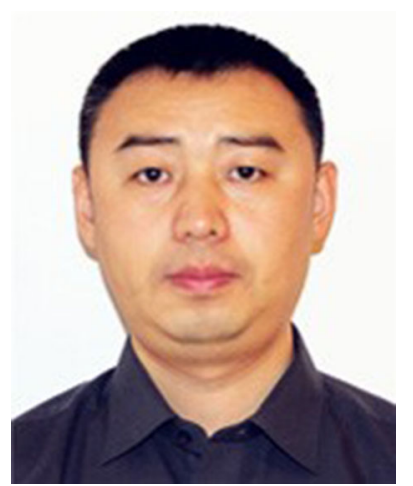

Chengyan Li is currently a professor in the School of Computer Science and Technology at Harbin University of Science and Technology, China. He received his bachelor's degree (1994) from Jilin University of Technology, and master's degree (1999) from Harbin University of Science and Technology. He has published more than 20 publications in international journals and conference proceedings, and has been invited to give keynote speeches at international conferences. His research interests include Data Mining, Clouding Computing, and Intelligent Information Processing.

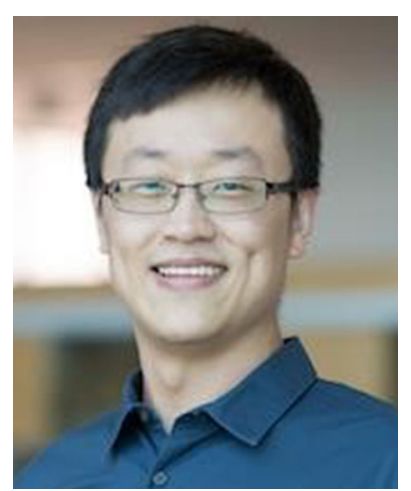

Xin Gao is a professor of computer science in Computer, Electrical and Mathematical Sciences and Engineering Division at King Abdullah University of Science and Technology (KAUST). He is also the Acting Associate Director of the Computational Bioscience Research Center, Deputy Director of the Smart Health Initiative, and the Lead of the Structural and Functional Bioinformatics Group at KAUST. Prior to joining KAUST, he was a Lane Fellow at Lane Center for Computational Biology in School of Computer Science at Carnegie Mellon University. He earned his bachelor degree in Computer Science in 2004 from Tsinghua University and his Ph.D. degree in Computer Science in 2009 from University of Waterloo. His research interest lies at the intersection between computer science and biology. In the field of computer science, he is interested in developing machine learning theories and methodologies related to deep learning, probabilistic graphical models, kernel methods and matrix factorization. He has published more than 230 papers in the fields of bioinformatics and machine learning. He is the associate editor of Genomics, Proteomics \& Bioinformatics, BMC Bioinformatics, Journal of Bioinformatics and Computational Biology, and Quantitative Biology, and the guest editor-in-chief of IEEE/ACM Transactions on Computational Biology and Bioinformatics, Methods, and Frontiers in Molecular Bioscience.

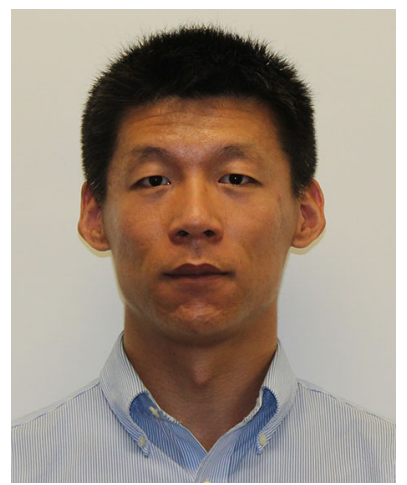

Qingquan Sun is an associate professor in the School of Computer Science and Engineering at California State University San Bernardino, USA. He received the Ph.D. degree in Electrical and Computer Engineering at The University of Alabama, Tuscaloosa, AL, USA in 2013. Dr. Sun has published around 40 journal/conference papers and book chapters. He also has led 3 NSF projects. Dr. Sun's research has been supported by U.S. National Science Foundation, U.S. National Institute of Health, and other sources. His research interests include intelligent sensing, machine learning and mobile computing in cyber physical systems. Dr. Sun is internationally recognized in intelligent sensing and machine learning fields. He has served as an associate editor for three international journals, a chair for an international conference, and a reviewer for around 20 international journals. 